\title{
Determining the $\beta$ Function of the Strong Interaction Coupling and Closing the Light Gluino Window
}

\author{
F. Csikor ${ }^{1}$ and Z. Fodor ${ }^{2} *$ \\ ${ }^{1}$ Institute for Theoretical Physics, Eötvös University, H-1088 Budapest, Hungary \\ ${ }^{2}$ Theory Division, CERN, CH-1211 Geneva 23, Switzerland \\ (Received 13 November 1996; revised manuscript received 24 March 1997)
}

\begin{abstract}
We discuss the perturbative running of $\alpha_{s}$ in a model independent way. Our analysis contains data on the hadronic $\tau$ decay and hadronic cross sections in $e^{+} e^{-}$annihilation between $5 \mathrm{GeV}$ and $M_{Z}$. We determine the color coefficients and the perturbative $\beta$ function of the strong interaction. The results are in agreement with QCD and rule out the QCD + light gluino scenarios on the 70.8\%93.0\% C.L. We combine our method with that of the multijet analysis at LEP. The combined result rules out light gluinos on the $99.76 \%$ C.L., provided that nonperturbative effects are not large. [S0031-9007(97)03330-9]
\end{abstract}

PACS numbers: 12.38.Qk, 11.10.Hi, 12.60.Jv, 13.65.+i

Asymptotic freedom is one of the most interesting predictions of QCD. In order to study the running of $\alpha_{s}$, one can collect its values at different scales (e.g., $\tau$ decay, deep inelastic scattering (DIS), $\Upsilon$ decay, $e^{+} e^{-}$) and compare them [1]. Another way is to analyze a single experiment (e.g., $\bar{p} p, e p$, or $\tau$ decay [2]). Nice agreement has been found between experiments and QCD. Some differences between the results obtained from lowenergy and high-energy experiments led to a number of speculations; e.g., that the apparent slower running of $\alpha_{s}$ could be due to additional light fermions of the theory.

Supersymmetric phenomenology deals normally with sparticles of masses $\mathcal{O}(100 \mathrm{GeV})$. The only exception is the light gluino [3] with mass $\leq 1.5 \mathrm{GeV}$ (window I) [4,5] and 3-5 GeV (window III) [6]. We will discuss window I and window III separately. Since such a light particle influences the running of $\alpha_{s}$ the comparison of high- and low-energy experiments could open or close the light gluino window [7-9]. A consistent analysis must contain the virtual gluino effects not only in the running (technically in the $\beta$ function), but in all loop diagrams, extracting $\alpha_{s}$ from the experimental results, too [9].

One can even determine the $\beta$ function of the strong interaction as done in Ref. [2]. In order to extract the three-loop coefficients one needed large values of $\alpha_{s}$, that is, the small energies of $\tau$ decay. The result is in good agreement with QCD.

It has been suggested [10] to look for gluinos in fourjet events. The LEP Collaborations determined the color coefficients $\left(C_{A} / C_{F}\right.$ and $\left.T_{F} / C_{F}\right)$ in multijet events. The results (cf. [1]) are in good agreement with QCD. An extension of QCD including light gluinos would result in a somewhat different $T_{F} / C_{F}$. The four-jet cross section is known only at tree level. Without the knowledge of the loop corrections this method alone can hardly give a clear answer to the question of light gluinos.

In this Letter we determine the color coefficients and the perturbative $\beta$ function of the strong interaction from experimental results. We consider gauge theories with fermions in the fundamental representation and their extended versions with light gluinos. We perform an analysis on the three-loop level in the $\overline{M S}$ scheme. The experimental inputs are $R_{\tau}$ and $R_{h}$ for energies between $5 \mathrm{GeV}$ and $M_{Z}$. We combine our method with that of the multijet analysis at LEP.

One of the clearest ways to study the running of the coupling, thus the $\beta$ function, of the theory would be to study one given experimental quantity at different energies. We chose $R_{h}$ 's at energies larger than $5 \mathrm{GeV}$, which are very clear quantities both experimentally and theoretically, due to their minimal nonperturbative corrections. Moreover, these are the only quantities known to $\mathcal{O}\left(\alpha_{s}^{3}\right)$ relative to the leading order, thus only a minimal scale ambiguity is present. In order to study the running of $\alpha_{s}$ one needs precise $R_{h}$ measurements for a large energy region. Unfortunately, $R_{h}$ measurements are limited by their small statistics for energy scales below $M_{Z}$. For this reason we have also considered another, strongly related (still not very low scale) quantity $R_{\tau}$ in our analysis. Since we are aware of the theoretical criticism on $\alpha_{s}$ determinations based on $R_{\tau}$ we include the effects of all known uncertainties in case of QCD [11-13]. The nonperturbative estimates to $R_{\tau}$ in the light gluino case might be unreliable. Therefore, we do not include $R_{\tau}$ in our analysis for window I gluinos. (Window III gluinos are much too heavy to contribute to $R_{\tau}$.)

Let us suppose that the strong interaction is described by a gauge theory based on a simple Lie group, which fixes the color coefficients of the theory $\left(C_{F}, C_{A}, T_{F}\right)$. The three basic processes in the theory are gluon bremsstrahlung from a quark, splitting of a gluon into two gluons, and splitting of a gluon into two quarks. To lowest order their amplitudes are proportional to a universal coupling and to $C_{F}, C_{A}$, and $T_{F}$, respectively. For example, for an $\mathrm{SU}(N)$ gauge group one has $C_{F}=\left(N^{2}-1\right) / 2 N, C_{A}=N$, and $T_{F}=1 / 2$. The $\beta$ function of the strong interaction, hadronic cross sections and widths are calculated in terms of these coefficients and the active number of fermions. We will look for a set of parameters which describes the experimental 
data most accurately and give the corresponding confidence level (C.L.) regions. The outcome-that is the best fit - does not necessarily predict a meaningful theory. Nevertheless, it could tell the difference between QCD and $\mathrm{QCD}+$ light gluino scenario.

The $\beta$ function: $d a(\mu) / d \ln \mu=-\beta_{0} a^{2}-\beta_{1} a^{3}-\beta_{2} a^{4}$ is known up to three-loop order. We factorize out the inconvenient $\pi$ s by using $a=C_{F} \alpha_{s} / 2 \pi$. Introducing $x=$ $C_{A} / C_{F}$ and $y=T_{F} / C_{F}$ gives $\beta_{0}=11 x / 3-4\left(n_{f} y+\right.$ $\left.x n_{\tilde{g}} / 2\right) / 3$, where $n_{f}$ is the number of active flavors and $n_{\tilde{g}}$ is the number of active gluinos. The expressions for $\beta_{1}$ and $\beta_{2}$ are more complicated [14,15]. In the running of $\alpha_{s}$ we follow Ref. [16] for threshold effects. We solve the above renormalization group equations exactly.

The other important quantity in the analysis-the hadronic cross section in $e^{+} e^{-}$annihilation via a virtual photon - is known on the three-loop level, too.

$$
\begin{aligned}
R_{\gamma} & =\sigma\left(e^{+} e^{-} \rightarrow \gamma \rightarrow \text { hadr }\right) / \sigma_{o}\left(e^{+} e^{-} \rightarrow \gamma \rightarrow \mu \mu\right) \\
& =3 \Sigma_{f} q_{f}^{2}\left(1+K_{1} a+K_{2} a^{2}+K_{3} a^{3}+\ldots\right)
\end{aligned}
$$

where $K_{1}=3 / 2$. $K_{2}, K_{3}$ is more complicated and also known for arbitrary $C_{F}, C_{A}$, and $T_{F}$ [14,17]. The hadronic (hadr) decay ratio of the $\tau$ is defined $R_{\tau}=\Gamma\left(\tau^{-} \rightarrow \nu_{\tau}+\right.$ hadr) $/ \Gamma\left(\tau^{-} \rightarrow \nu_{\tau} e^{-} \bar{\nu}_{e}\right)$. Its perturbative value is strongly related to $R_{\gamma}$. For a recent analysis and a review on $R_{\tau}$ see, e.g., [12] and [18]. The hadronic decay width of the $Z$ boson $R_{Z}=\Gamma(Z \rightarrow \operatorname{hadr}) / \Gamma\left(Z \rightarrow \mu^{+} \mu^{-}\right)$is again a function of $R_{\gamma}$ and known at the three-loop level. At the energy scales of the analysis important electroweak and mixed corrections appear. We include these corrections.

The experimental values for $R_{\tau}$ are given in [19] with an average of $R_{\tau}=3.616 \pm 0.02$. Averaging for the four LEP experiments and three leptons [20] gives $R_{Z}=20.778 \pm$ 0.029 . The references for hadronic cross sections at energies below $M_{Z}$ are taken from [21]. We have collected all the existing published data, and some unpublished results, too. Some of them were binned by the experimental groups. The total number of data points included in our analysis is 182 .

Combining results from different experiments is a delicate question, with major problems. (i) The use of the radiative corrections is not unique, reflecting the state of art at the time of publication. (ii) The results do depend on the mass of the $Z$ boson and top quark- which was assumed to be approximately $20 \mathrm{GeV}$ at the early eighties. (iii) The measurement of the total cross section was not performed in the full phase space, and Monte Carlo acceptance calculations based on different assumptions were done. We corrected for the first two problems; correction for the third one is practically impossible. For further details see $[22,23]$.

There are different sources of uncertainties in the determination of $\alpha_{s}$. We treat them in a unified manner. We add the systematic errors linearly. The total systematic error estimates and the statistical errors are combined quadratically. The overall normalization errors within one experiment are correlated. We include these correlations.
We minimize $\chi^{2}=\Delta^{T} V^{-1} \Delta$, where $\Delta$ is an $n$ vector of the residuals of $R_{i}-R_{\mathrm{fit}}$ for the $n$ individual results and $V$ is an $n \times n$ error matrix. In $V$, the diagonal elements $V_{i i}$ are the squares of the total errors for the $i$ th measurement and the off-diagonal elements $V_{i j}$ correspond to the correlations between the $i$ th and $j$ th measurements. For different points from the same experiment $V_{i j}$ is given by the product of the normalization errors [22]. The separation of the systematics into point-to-point and overall normalization error is ambiguous. Our results are rather insensitive to a moderate change of this splitting in a given experiment. For some experiments the above separation was not even explicitly given. We checked that our results are stable against even large variations of the splitting in these cases. However, the unreasonable extreme case of totally uncorrelated fitting would give rather different results with much more predictive power. We have assumed that the results of different experiments are uncorrelated. In order to perform a further consistency check we included an additional hypothetical overall error of $1 \%$ in the correlation matrix. The change of the result turned out to be negligible. Since the experimental groups usually give their results with binning in fixed energy intervals, we checked that further binning had practically no influence on our results.

Because of the relatively poor statistics for the $e^{+} e^{-}$ experiments below $M_{Z}$ the theoretical uncertainties are dominant only for $R_{\tau}$ and non-negligible for $R_{Z}$. Estimating the nonperturbative and perturbative errors in $\tau$ decay we use the results of $[11,12]$. For a fixed order QCD calculation we assume that the error is equal to the last computed term. Including all the errors we get $R_{\tau}=3.616 \pm 0.143$, which corresponds to $\alpha_{s}\left(M_{\tau}\right)=$ $0.335 \pm 0.053$ and $R_{Z}=20.778 \pm 0.0387$, which corresponds to $\alpha_{s}\left(M_{Z}\right)=0.123 \pm 0.006$. We assume that currently incalculable and/or model dependent nonperturbative corrections are negligible or correctly estimated. If the nonperturbative corrections turn out to be larger than our estimates, the confidence levels for light gluino exclusion to be determined below should be lowered. Note that both error estimates are certainly very conservative.

Having included all the errors one can determine the best fit values and the C.L. regions for $x=C_{A} / C_{F}$ and $y=T_{F} / C_{F}$ with or without light gluinos.

Figure 1 contains the $68.3 \%$ C.L. regions for theories without gluinos and with window III gluinos. As it can be seen the experimental results are in agreement with QCD; however, they rule out the QCD + light gluino scenario on the 93 (90.7)\% C.L.'s for $M_{\tilde{g}}=3(5) \mathrm{GeV}$, respectively. The variation of the fixed $\chi^{2}$ boundaries is quite small for the above mass region. For binning level 50 (d.o.f. 48) the $\chi^{2}$ values are 37.37 without and 37.94 with gluinos $\left(M_{\tilde{g}}=3 \mathrm{GeV}\right)$ for the best fits. As mentioned before, for window I gluinos we do not include $R_{\tau}$ in our study. In this case we have a $70.8 \%$ exclusion only.

Note the important difference between the presentations of our result and that of the four-jet analysis for the case with light gluinos [1]. In our case the best fits and the 


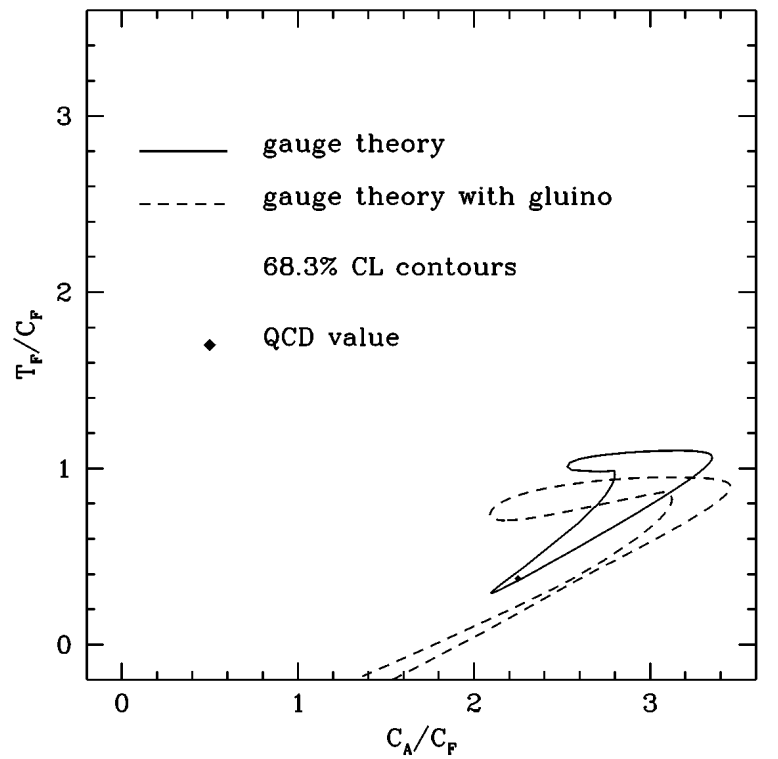

FIG. 1. The $68.3 \%$ C.L. regions for $x=C_{A} / C_{F}$ and $y=$ $T_{F} / C_{F}$. The QCD value is represented by a diamond.

C.L. contours for the $x, y$ variables are determined separately for the theories with and without light gluinos. These results are compared for both theories with the QCD values: $\bar{x}=2.25, \bar{y}=0.375$. Because of the simplicity of the tree-level treatment of the four-jet analysis in Ref. [1], it was possible to produce the same best fits and contours for both theories and compare this unique result with $\bar{x}, \bar{y}$ above in the theory without light gluinos, and with $\bar{x}$ and with an effective $\bar{y}_{\text {eff }}=0.6$ for the gluino extended theory. The four-jet results can easily be expressed in terms of our variables.

Two remarks are in order: (i) The four-jet analysis is based on tree-level calculations, whereas our method contains corrections up to three loops. (In [1] also the oneloop analysis to 2 and 3 jets is done; however, the errors on $x, y$ are huge.) (ii) Our method contains the running of $\alpha_{s}$, too, thus it gives a simultaneous check of the $\beta$ function.

Assuming [24] that the underlying gauge group is $\mathrm{SU}(3)$, we may consider the number of gluinos as a free parameter. The best fits give $n_{\tilde{g}}=-0.638 \pm 1.17$ for window I and $n_{\tilde{g}}=0.0078 \pm 0.52$ for window III. The C.L.'s are given by Bayes's theorem allowing only non-negative integer $n_{\tilde{g}}: 72.5 \%$ for window I and $87.7 \%$ for window III. (For modeling data with bounded physical region, $n_{\tilde{g}} \geq 0$, and for application of Bayes's theorem see, e.g., Sec. 28 of [21].) Finally, one can also fix the number of gluinos and determine $\alpha_{s}\left(M_{Z}\right)$. For $n_{\tilde{g}}=0$ (i.e., QCD) we get $0.124 \pm 0.004$, for $n_{\tilde{g}}=1$ we get $0.129 \pm 0.006$ (window I) and $0.132 \pm 0.006$ (window III). As expected [21] the QCD value is slightly larger than the world average.

Figure 2 shows the $\beta$-function coefficients with $68.3 \%$ and $95.4 \%$ C.L. regions for models without gluinos at $M_{Z}$. These curves represent merely a transformation of the curves in the $(x, y)$ plane; they result in similar conclusions for QCD. As it can be seen the value of $\beta_{0}$ is quite well constrained; however, not even the sign of

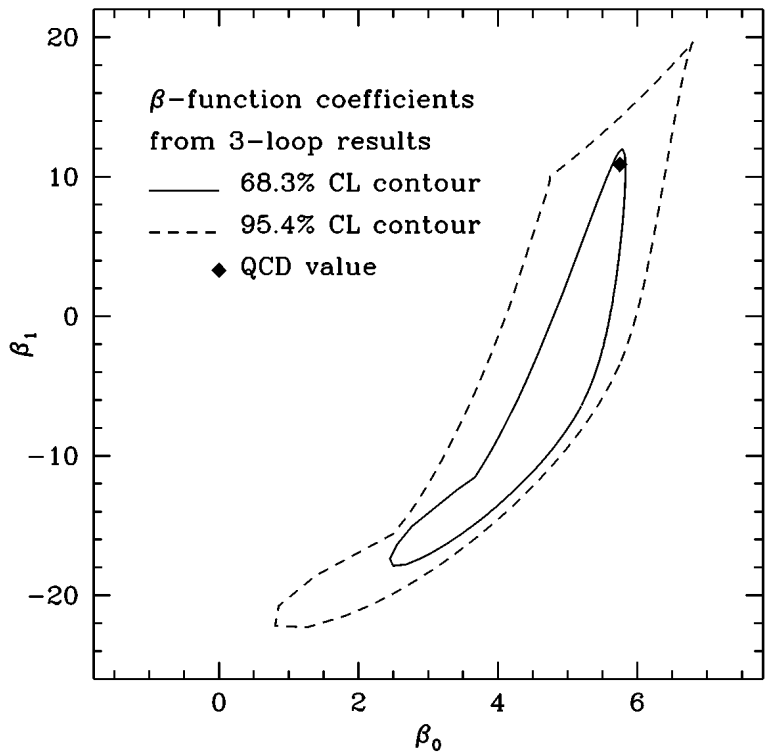

FIG. 2. The $68.3 \%$ and $95.4 \%$ C.L. regions for $\beta_{0}$ and $\beta_{1}$.

$\beta_{1}$ could be predicted. This result reflects the fact that in the energy region studied the running of the coupling is determined almost exclusively by the one-loop term, proportional to $\beta_{0}$. An interesting possibility is to fix the cross sections, $\beta_{1}, \beta_{2}$, and the flavor dependence of $\beta_{0}$ to their perturbative QCD values and extract $\beta_{0}$ from the fits. The result is $\beta_{0}=5.70 \pm 0.64$ to be compared with the theoretical value of 5.75. Following [2] we fixed $\beta_{0}$ and $\beta_{1}$ to their theoretical (QCD) value and performed a fit with $\beta_{2}$ as a free parameter. The allowed region for $\beta_{2}$ contains the theoretical prediction, but the error is an order of magnitude larger than the theoretical value.

Comparing our Fig. 1 and Fig. 10 of [1] - taking proper care of using the same variables - one observes that the overlap of the $68.3 \%$ C.L. regions for the two analyses is quite small. This opens the possibility for a much stronger restriction on the theories without and with gluino. We have parametrized the $\chi^{2}$ of the multijet analysis as a function of $x$ and $y$, and performed a new fit adding it to our $\chi^{2}$ function. In the actual analysis we have included the new results in [25]. Since the four-jet analysis is based on a tree-level calculation we have included the unknown higher order QCD and mass effects. We assumed much larger uncalculated higher order corrections than used in the experimental papers. Because of them we enlarged the axes of the error ellipse by $12 \%$ of the theoretical $x$ and $y$ values [relative correction of $\mathcal{O}\left(\alpha_{s}\right)$ ]. For the fourjet analyses we have used the mass effects calculated by [26]. The results of the combined analysis for QCD and window III gluinos are presented in Fig. 3. Our result is consistent with QCD + no gluino scenario, while the theory with window III light gluino is excluded on the 99.99(99.89)\% C.L. for $M_{\tilde{g}}=3(5) \mathrm{GeV}$. The window I gluino is excluded on the $99.97 \%$ C.L.

Again one can fix the underlying gauge group to $\mathrm{SU}(3)$ and consider the number of gluinos as a parameter to 


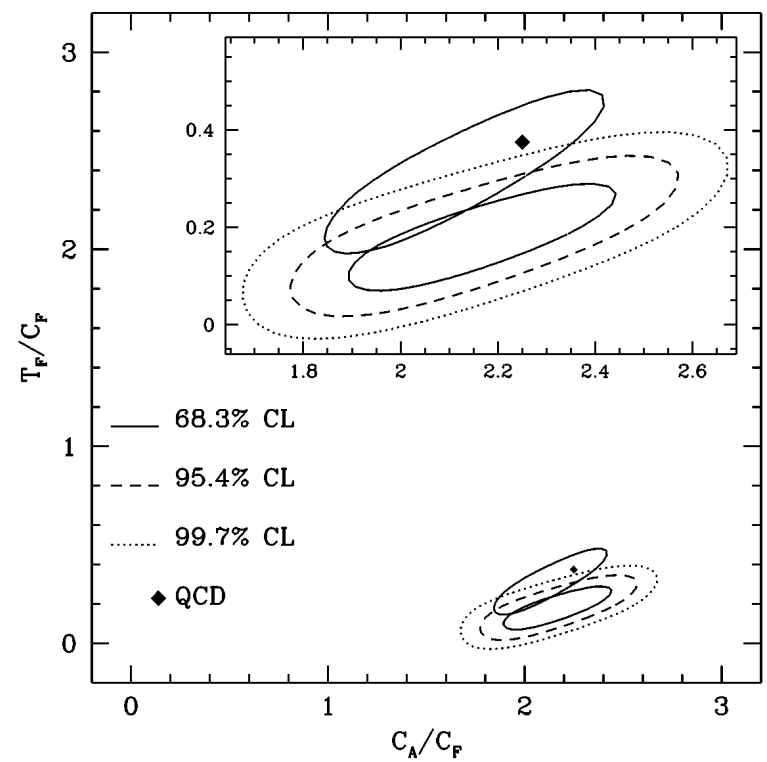

FIG. 3. The $68.3 \%, 95.4 \%$, and $99.7 \%$ C.L. regions of the combined analysis for the theory with window III light gluino $\left(M_{\tilde{g}}=3 \mathrm{GeV}\right)$ and the $68.3 \%$ C.L. region for the theory without gluino.

be fitted. We get $n_{\tilde{g}}=-0.35 \pm 0.33$ for window I and $n_{\tilde{g}}=-0.156 \pm 0.27\left(n_{\tilde{g}}=-0.197 \pm 0.32\right)$ for window III $M_{\tilde{g}}=3(5) \mathrm{GeV}$ gluinos. Using Bayes's theorem assuming that $n_{\tilde{g}}$ is a non-negative integer number, the exclusion C.L.'s are $99.96 \%$ for window I and $99.96(99.76) \%$ for window III gluinos.

Leaving out $R_{\tau}$ from the window III combined analyses, the C.L. values change only negligibly.

In summary, we have presented a method to analyze the running of $\alpha_{s}$ from $M_{\tau}$ to $M_{Z}$. We have determined the group coefficients of the theory, which are in agreement with QCD. The $\beta$-function coefficients are also given with a similar result. Furthermore, our results rule out the QCD + light gluino scenario on the $70.8 \%$ and $90.7 \%-93.0 \%$ C.L. for windows I and III, respectively. Combining the analysis of the present Letter and the method using multijet data (cf. Ref. [1,25], however, treating the theoretical errors more conservatively than the experimental groups) we have got an order of magnitude smaller C.L. regions for the allowed group coefficients, which supports QCD within $1 \sigma$ and excludes the QCD + light gluino scenario with a 99.97\% C.L. for window I and at least $99.89 \%$ C.L. for window III gluinos. Performing a one parameter fit to determine the number of gluinos, we get $n_{\tilde{g}}=-0.35 \pm 0.33$ and $n_{\tilde{g}}=-0.197 \pm 0.32$ for windows I and III, respectively. In terms of C.L.'s this corresponds to $99.96 \%$ and $99.76 \%$. Needless to say, our results are valid assuming the light gluino extension of the standard model. We have not investigated whether additional new physics could change our exclusion C.L. limits.

The details of the above analysis, the convergence features, and the statistical analysis will be presented in a forthcoming publication [27].
We thank M. Drees, B. Gary, A.L. Kataev, and R. Sommer for discussions. This work was partially supported by Hung. Sci. Grants No. OTKA-T16248 and No. T22929.

*On leave from Institute for Theoretical Physics, Eötvös University, H-1088 Budapest, Hungary.

[1] M. Schmelling, in Proceedings of the 15th International Conference on Physics in Collision (Editions Frontieres, Gif-Sur-Yvette, 1995), p. 287.

[2] M. Girone and M. Neubert, Phys. Rev. Lett. 76, 3061 (1996).

[3] G. Farrar and P. Fayet, Phys. Lett. B 76, 575 (1978).

[4] G. Farrar, Phys. Rev. D 51, 3904 (1995).

[5] C. Clavelli and P.W. Coulter, Phys. Rev. D 51, 1117 (1995).

[6] M. B. Cakir and G. Farrar, Phys. Rev. D 50, 3268 (1994).

[7] I. Antoniadis, J. Ellis, and D. V. Nanopoulos, Phys. Lett. B 262, 109 (1991).

[8] M. Jezabek and J. H. Kühn, Phys. Lett. B 301, 121 (1993).

[9] J. Ellis, D. V. Nanopoulos, and D. A. Ross, Phys. Lett. B 305, 375 (1993); M. Schmelling and R. D. St. Denis, Phys. Lett. B 329, 393 (1993).

[10] B. A. Campbell, J. Ellis, and S. Rudaz, Nucl. Phys. B198, 1 (1982).

[11] G. Altarelli, P. Nason, and G. Ridolfi, Z. Phys. C 68, 257 (1995).

[12] M. Neubert, Nucl. Phys. B463, 499 (1996).

[13] M. Shifman, Int. J. Mod. Phys. A 11, 3195 (1996).

[14] S. G. Gorishni, A. L. Kataev, and S. A. Larin, Phys. Lett. B 259, 144 (1991); L. R. Surguladze and M. A. Samuel, Phys. Rev. Lett. 66, 560 (1991); 66, 2416(E) (1991).

[15] L. Clavelli, P. W. Coulter, and L. R. Surguladze, Phys. Rev. D 55, 4268 (1997).

[16] W. Bernreuter and W. Wetzel, Nucl. Phys. B197, 228 (1982).

[17] L. J. Clavelli and L. R. Surguladze, Phys. Rev. Lett. 78, 1632 (1997); K. G. Chetyrkin, Phys. Lett. B 391, 402 (1997).

[18] A. Pich, Nucl. Phys. (Proc. Suppl.) B39, 326 (1995).

[19] L. Duflot, Nucl. Phys. (Proc. Suppl.) B40, 37 (1995); T. Coan et al., Phys. Lett. B 356, 580 (1995).

[20] LEP Electroweak Working Group, Report No. LEPEWWG 96-02.

[21] Particle Data Group, R. M. Barnett et al., Phys. Rev. D 54, 1 (1996).

[22] H. J. Behrend et al., Phys. Lett. B 183, 400 (1987).

[23] G. D'Agostini, W. de Boer, and G. Grindhammer, Phys. Lett. B 229, 160 (1989); D. Haidt, in Directions in High Energy Physics, edited by P. Langacker (World Scientific, Singapore, 1995), Vol. 14, p. 203; S. Eidelman and F. Jegerlehner, Z. Phys. C 67, 585 (1995).

[24] A. de Gouvea and H. Murayama, Report No. hep$\mathrm{ph} / 9606449$.

[25] R. Barate et al., Report No. CERN-PPE-97-002.

[26] R. Munoz-Tapia and W. J. Stirling, Phys. Rev. D 49, 3763 (1994).

[27] F. Csikor and Z. Fodor (to be published). 